Thorax (1975), 30, 266.

\title{
Effect of preservation on the elasticity of human aortic valve homografts
}

\author{
Y. L. NG a nd J. E. C. W R I G H T \\ Department of Surgery, Cardiothoracic Institute, London Chest Hospital, London E2 9JX
}

\begin{abstract}
Ng, Y. L. and Wright, J. E. C. (1975). Thorax, 30, 266-270. Effect of preservation on the elasticity of human aortic valve homografts. The elastic properties of preserved human aortic homografts after different storage times in antibiotic-nutrient medium solution have been measured. There was a definite loss of elasticity within nine months leading to a rapid decrease after a year of preservation. It is likely that satisfactory valve function depends on its elasticity, and these studies indicate that aortic valves which have been exposed to antibiotic media for more than nine months should not be used in valve replacement surgery.
\end{abstract}

Since the introduction of aortic homografts more than 20 years ago, the replacement of diseased aortic valves with preserved aortic homografts has become an accepted procedure. The long-term patient survival results have been encouraging, and were reported by Karp and Kirklin in 1969 to be superior to that using mechanical prostheses. Aortic homografts are obtained from cadaver hearts within a few hours of death. Methods of valve preservation include freeze-drying, gamma irradiation, and chemical sterilization. Preservation by these methods has led to rupture and calcification of the valve tissues (Gross, 1954; Sauvage and Wesolowski, 1955; Creech et al., 1956; Shucksmith, Mitchell, and Zinnemann, 1958; Foster et al., 1965; Smith, 1967; Ross, 1967a; Brock, 1968). For these reasons antibiotic-nutrient medium sterilization has been developed and advocated. It has been reported that aortic valves so treated have retained their normal histological structure (Smith, 1967; Stinson et al., 1968; Barratt-Boyes and Roche, 1969; Lockey et al., 1972) and viable cells are found after several weeks (Al-Janabi et al., 1972). Preservation of the normal mechanical properties of the cusps must be aimed for, to ensure satisfactory function in the patient. Knowledge of the effect on the valve of the preparation method is essential, particularly if the valve is to be preserved for a long period before use. No reference could be found in the literature to changes in the elastic properties of antibioticsterilized homografts.

The aim of this study was to document the mechanical properties of the homograft valve tissue after preservation in antibiotic-nutrient medium solution for different periods of time.

\section{METHODS}

Aortic valves were dissected from cadaver hearts $\stackrel{\circ}{\varnothing}$ of either sex within 72 hours of death. The valves $\overrightarrow{\vec{\theta}}$ were preserved and stored in a freshly prepared 3 antibiotic-nutrient medium solution (Lockey et al., 1972) kept at a constant temperature of $4^{\circ} \mathrm{C}$. Immediately before each study the valves were washed with distilled water, and wherever possible응 each of the three cusps was in turn subjected to $\stackrel{\otimes}{x}$ stress by the method reported previously (Wright and $\mathrm{Ng}, 1974)$. Wright and $\mathrm{Ng}$ (1974) demon-3.3. strated that for a fresh aortic valve the elastic $\delta$ properties of the three cusps, when subjected to 3 stress, were similar, with no significant difference 을 between them. In the present study the results from separate cusps of the same valve were을 averaged. The cusp tissue was securely sandwiched between two serrated steel rings, each of which $\%$ has a central diameter aperture of $10 \mathrm{~mm}$. The $N$ steel rings were fastened by screws to a steel pressure chamber which was sealed with a thin gasket. The pressure in the system was raised by injecting distilled water from a constant volume pump. An $\frac{\widetilde{\Phi}}{\Phi}$ outlet from the steel chamber was connected to $\stackrel{?}{?}$ a pressure transducer (Bell and Howell Type 0 4-327-L221), which measured directly the pressure $\stackrel{0}{P}$ variation in the chamber. The transducer signal $\stackrel{\Phi}{\stackrel{D}{\circ}}$ was registered by a Gould Brush 400 pen recorder $\mathbb{D}$ moving at a constant speed of $5 \mathrm{~mm}$ per second.

The whole system was flushed out with carbon dioxide and assembled underwater to eliminate 
all trace of air. If any air remains in the system, the resultant pressure-volume curves are invalid. Any slackness in the specimen was overcome by applying a slight initial pressure of $0.133 \mathrm{kPa}$ $(1 \mathrm{mmHg})$. This represented the starting pressure in all cases. When the pressure was next applied by the constant volume pump, the full force would act on the specimen, and the extension of the cusp tissue would depend on, and reflect, its elasticity. The initial pressure of $0.133 \mathrm{kPa}(1 \mathrm{mmHg})$ had been taken into account in the calculations. The specimens were tested at room temperature, and for each specimen as near as possible the central part of the cusp tissue was used. A steel blank was used to measure the inherent elasticity of the system. The pressure-volume curve obtained from the blank reflected the degree of error in the system, and was also allowed for in all calculations.

A total of 41 cusps was studied. They were from adult hearts (30 to 65 years of age). They had been preserved and kept in the storage solution for from one month to a year previously.

For all specimens a series of pressure-volume curves was obtained, and the results were averaged. The difference between sequential curves was less than $\pm 2.0 \%$.

\section{RESULTS}

To calculate a true modulus of elasticity would necessitate conversion of the pressure-volume curves to tension-elongation curves, as was done in a previous paper (Mundth, Wright, and Austen, 1971). However, as was shown in that paper and by Wright and $\mathrm{Ng}$ (1974), the two curves are directly proportional to each other, and we feel that conversion is not necessary. We prefer to use an incremental measurement of elasticity described previously (Wright and $\mathrm{Ng}, 1974$ ). Since pressure is directly proportional to stress, and volume to strain, changes in volume reflect changes in elasticity.

Two measurements are made. The first is the volume injected between $0 \cdot 133$ and $13.3 \mathrm{kPa}(1$ and $100 \mathrm{mmHg}$ ). This represents the overall elastic response for that change in pressure. The second measurement is the volume of fluid injected over the first increment of $1.66 \mathrm{kPa}(12.5 \mathrm{mmHg})$, i.e., $0.133-1.66 \mathrm{kPa}(1-12.5 \mathrm{mmHg})$. We have shown that the cusp is elastic over this early pressure change and then becomes relatively inelastic when the pressure exceeds about $1.99 \mathrm{kPa}(15 \mathrm{mmHg})$. Figure 1 demonstrates this property in a cusp preserved for one month.

Valves preserved for up to nine months produce pressure-volume curves which show no

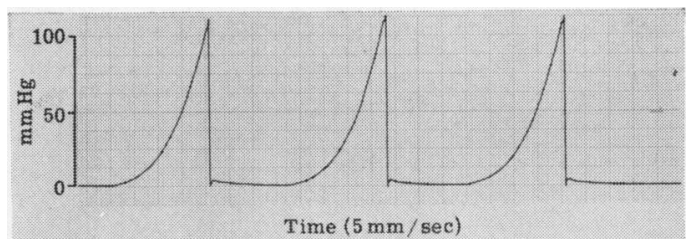

FIG. 1. Pressure-volume curves for antibiotic-nutrient preserved human aortic valve cusp.

statistical significant difference at the $95 \%$ level when compared to curves from fresh valves. An example is shown (Fig. 2) which is the pressurevolume curve from a valve preserved for eight and a half months compared to a curve from a fresh cusp.

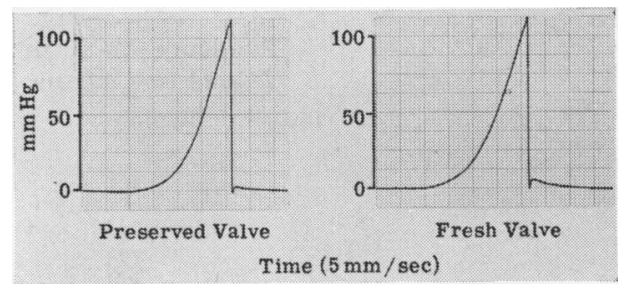

FIG. 2. Pressure-volume curves for antibiotic-nutrient preserved and fresh human aortic valve cusps.

Figure 3 shows a pressure-volume trace for a specimen which had been preserved for one year. The trace is compared again with one from a fresh cusp. The decrease in elasticity is apparent. The trace is more comparable to one produced from fascia lata, also shown in Figure 3. This latter trace is typical of tissue with poor elastic characteristics.
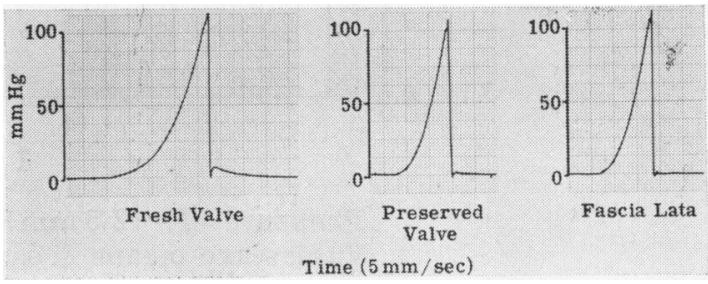

FIG. 3. Pressure-volume curves for fresh and antibiotic-nutrient preserved human aortic valve cusps and fresh fascia lata.

Figure 4 shows diagrammatically the reduction in overall elasticity as the homograft is preserved for longer periods. A paired Student's $t$ test was used to determine the statistical significance of the difference between the mean values of the elasti- 


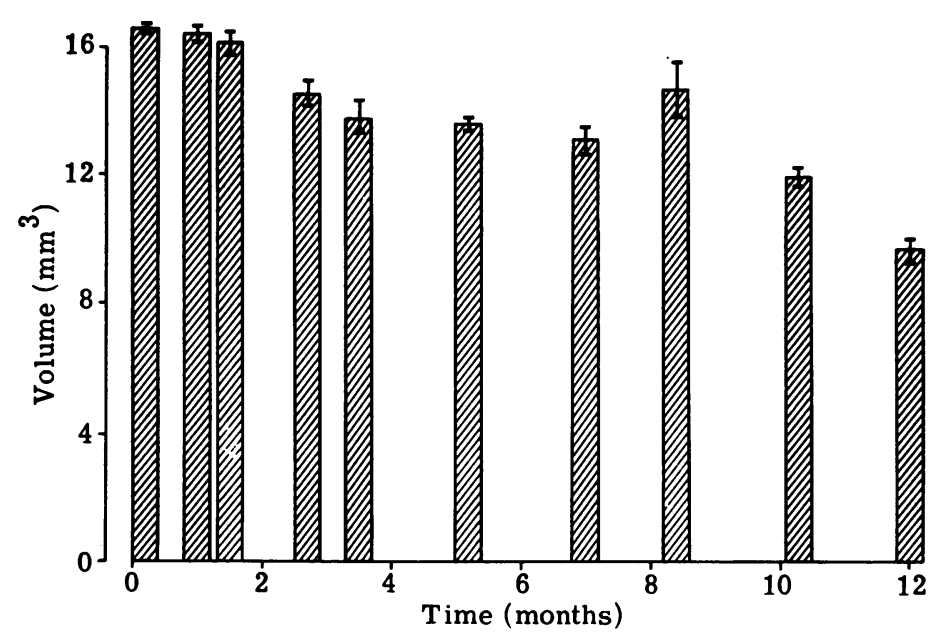

Pressure $=1-100 \mathrm{~mm} \mathrm{Hg}$

Values are means \& bars represent S.E.M. $(\mathrm{N}=3-6)$

FIG. 4. Elasticity of preserved human aortic valve cusps.

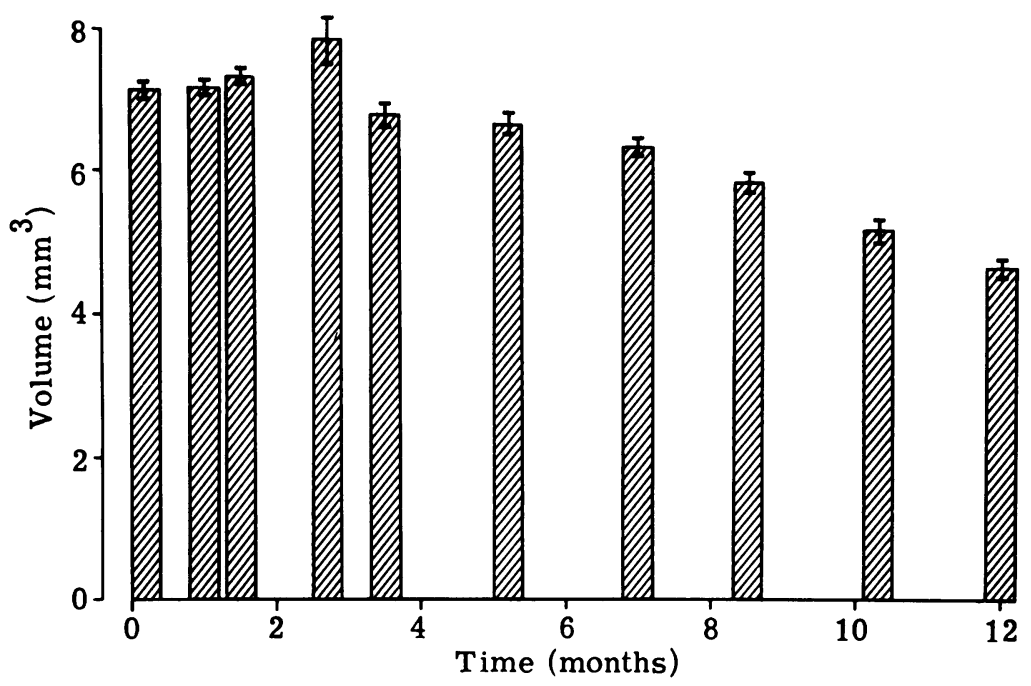

Pressure $=1-12.5 \mathrm{~mm} \mathrm{Hg}$

Values are means \& bars represent S.E. M. $(\mathrm{N}=3-6)$

FIG. 5. Elasticity of preserved human aortic valve cusps.

city between different periods of preservation. This showed that the $t$ value is not significant at the $95 \%$ level of confidence when the preservation is less than nine months. However, above nine months the $t$ value is significant at this level. Figure 5 shows that there is a steady loss of elasticity over the first pressure increment, be coming significant only after nine months.

DISCUSSION

We have shown for the normal and the preserveç 
human aortic valve cusp a mechanical elastic property. Although the number of specimens used was not large, this was compensated for by the good reproducibility of all curves and values. We have measured changes in elasticity over equal increments of pressure change, a method now accepted (Burton, 1954; Remington, 1963). This method of analysis also enables cusp tissue to be compared with other tissue (Krafka, 1939; Burton, 1954; Bergel, 1961).

The pressure-volume curves display a definite non-linear relation over the initial increase of pressure up to between 1.33 and $2.66 \mathrm{kPa}(10$ and $20 \mathrm{mmHg}$ ). There is then a stiff phase for pressures above that, the difference between the two phases being a tenfold change in elasticity. After the specimens have been exposed to antibiotic-nutrient medium for nine months, there is a steady loss of elasticity, which becomes statistically significant after nine months.

Elastin and collagen fibres are the principal components of the valve cusp. Bergel (1961) showed that the two phases are due to these fibres, elastin contributing to the early phase and collagen to the later stiff phase. When the aortic valve is preserved in an antibiotic-nutrient medium solution for some length of time, the preservation solution must have an effect on the elastin component of the fibre, thereby reducing its elasticity and making the cusp tissue stiffer.

It is probable that the cusp elastic property facilitates cusp function by allowing the gradual development of tension in the cusp during the closing phase of the valve, a factor which may prolong cusp life. Further, as the cusp stretches, the central part bulges. This reduces the radius of curvature, and hence the tension is reduced according to Laplace's Law. However, the tissue becomes stiff after the first increment of pressure, preventing prolapse of cusps, which could occur if the tissue remained equally elastic throughout its range. When a valve loses its elasticity due to antibiotic-nutrient medium solution preservation, it loses these properties and may not function properly. It may no longer be able to close completely to prevent retrograde flow. A valve which has lost its elastic property should not be used in valve replacement surgery. With these considerations in mind we propose that an aortic homograft which has been preserved in antibiotic-nutrient medium solution for more than nine months should be discarded, and although not reaching statistical significance, our results indicate that deterioration is evident at three months.
This work was supported by a grant from the National Heart and Chest Hospitals Clinical Research Commmittee.

\section{REFERENCES}

Al-Janabi, N., Gonzalez-Lavin, L., Neirotti, R., and Ross, D. N. (1972). Viability of fresh aortic valve homografts: a quantitative assessment. Thorax, 27, 83.

Barratt-Boyes, B. G. and Roche, A. H. G. (1969). A review of aortic valve homografts over a six and one-half year period. Annals of Surgery, 170, 483.

Bergel, D. H. (1961). The static elastic properties of the arterial wall. Journal of Physiology, 156, 445.

Brock, Lord (1968). Long-term degenerative changes in aortic segment homografts, with particular reference to calcification. Thorax, 23, 249.

Burton, A. C. (1954). Relation of structure to function of the tissues of the wall of blood vessels. Physiological Reviews, 34, 619.

Creech, O. Jr, DeBakey, M. E., Cooley, D. A., and Halpert, B. (1956). Structural alterations in human aortic homografts one to two and one-half years after transplantation. Surgery, Gynecology and Obstetrics, 103, 147.

Foster, J. H., Collins, H. A., Jacobs, J. K., and Scott, H. W. Jr (1965). Long-term follow-up of homografts used in the treatment of coarctation of the aorta. Journal of Cardiovascular Surgery, 6, 111.

Gross, R. E. (1954). In the discussion of a paper by Creech, O., DeBakey, M. E., Self, M., and Halpert, B. (1954). The fate of heterologous arterial grafts: an experimental study. Surgery, 36, 431 .

Karp, R. B. and Kirklin, J. W. (1969). Replacement of diseased aortic valves with homografts. Annals of Surgery, 169, 921.

Krafka, J. Jr (1939). Comparative study of the histophysics of the aorta. American Journal of Physiology, 125, 1.

Lockey, F., Al-Janabi, N., Gonzalez-Lavin, L., and Ross, D. N. (1972). A method of sterilizing and preserving fresh allograft heart valves. Thorax, 27, 398.

Mundth, E. D., Wright, J. E. C., and Austen, W. G. (1971). Development of a method for stress/ strain analysis of cardiac valvular tissue. Current Topics in Surgical Research, 3, 67.

Remington, J. W. (1963). The physiology of the aorta and major arteries. In: Handbook of Physiology. Circulation. Section 2: Volume II, Chapter 24, pp. 799-838. Edited by W. F. Hamilton, American Physiology Society, Washington, DC.

Ross, D. N. (1967a). Homograft replacement of the aortic valve. British Journal of Surgery, 54, 842. (1967b). Replacement of aortic and mitral valves with a pulmonary autograft. Lancet, 2, 956.

Sauvage, L. R. and Wesolowski, S. A. (1955). The healing and fate of arterial grafts. Surgery, 38, 1090. 
Shucksmith, H. S., Mitchell, W. M., and Zinnemann, K. (1958). Two and a half years' survival of a grafted abdominal aortic aneurysm with histological studies. British Journal of Surgery, 46, 141.

Smith, J. C. (1967). The pathology of human aortic valve homografts. Thorax, 22, 114.

Stinson, E. B., Angell, W. W., Iben, A. B., and Shumway, N. E. (1968). Aortic valve replacement with fresh valve homograft. American Journal of
Surgery, 116, 204.

Wright, J. E. C. and Ng, Y. L. (1974). Elasticity of $\frac{\bar{\sigma}}{\bar{c}}$ $\underset{R \text { hesearch, 8, 384. }}{\text { human aortic }}$

Requests for reprints to: J. E. C. Wright, F.R.C.S

Department of Surgery, Cardiothoracic Institute, London Chest Hospital, London E2 9JX. 